\title{
Flexural fracturing of a cohesive granular layer
}

\author{
Jean-Christophe Géminard, Lorène Champougny, Pierre Lidon and Francisco Melo ${ }^{\dagger}$ \\ Université de Lyon, Laboratoire de Physique, Ecole Normale Supérieure de Lyon, \\ CNRS, UMR 5672, 46 Allée d'Italie, 69007 Lyon, France. and \\ $\dagger$ Departamento de Física, Universidad de Santiago de Chile, \\ Av. Ecuador 3493, Casilla 30\%, Correo 2, Santiago, Chile.
}

\begin{abstract}
We report on the fracturing of cohesive granular materials subjected to a flexural deformation. A thin layer of glass beads or of flour is deposited on an unstretchable membrane to which flexion is imposed. We observe the formation of a periodic fracturing-pattern whose characteristics are discussed in comparison with results previously obtained for an in-plane stretching [PRL 105, 208001 (2010)]. In particular, at a given relative humidity, the wavelength is observed to depend linearly on the layer thickness but to be independent of the grain size although the smallest grains are clearly more cohesive.
\end{abstract}

PACS numbers: 45.70.Qj: Pattern formation in granular systems; 89.75.Kd: Pattern formation in complex systems; 62.20.mt: Structural failure of materials.

\section{INTRODUCTION}

A relevant feature of humid sand is "strain softening" due both to a decrease of the associated adhesion force when a single bridge elongates [1] and to a decrease in the overall number of bridges which collapse when excessively stretched [2]. This peculiar characteristic can be seen as responsible of the plasticity of cohesive granular materials under tension and provides some clues why structures made of humid sand, such as sand castles, break generally in a catastrophic manner. In a recent article [3], we explored the response of a horizontal thin layer of cohesive material to the simplest mode of deformation. An extensible membrane was the heart of a suitable system to impose a homogeneous, in-plane, stretching to the layer. It was shown that "strain softening " is responsible of the nearly periodic structure that develops, modulating the strain field in the layer along the pulling axis, as soon as the external deformation is turned on. The associated wavelength was observed to increase linearly with the layer thickness, to be almost independent of particle size and to be a linear function of the relative humidity.

In the present report, we explore the response of a cohesive granular layer to bending. We discuss similarities and differences with in-plane stretching. Even though the imposed deformations qualitatively differ one from the other, most of the features are present in both cases, showing the robustness of "strain softening" as the driving mechanism of the instability.

\section{EXPERIMENTAL SETUP AND PROTOCOL}

The experiment consists in imposing a flexural deformation at the base of a thin layer of a cohesive granular material. To do so, the grains are initially spread onto an unstretchable membrane to which the deformation is imposed.

The membrane, a thin paper band (width $5 \mathrm{~cm}$ and total length $50 \mathrm{~cm}$ ), lyes on a cylinder (radius $R$ ) which

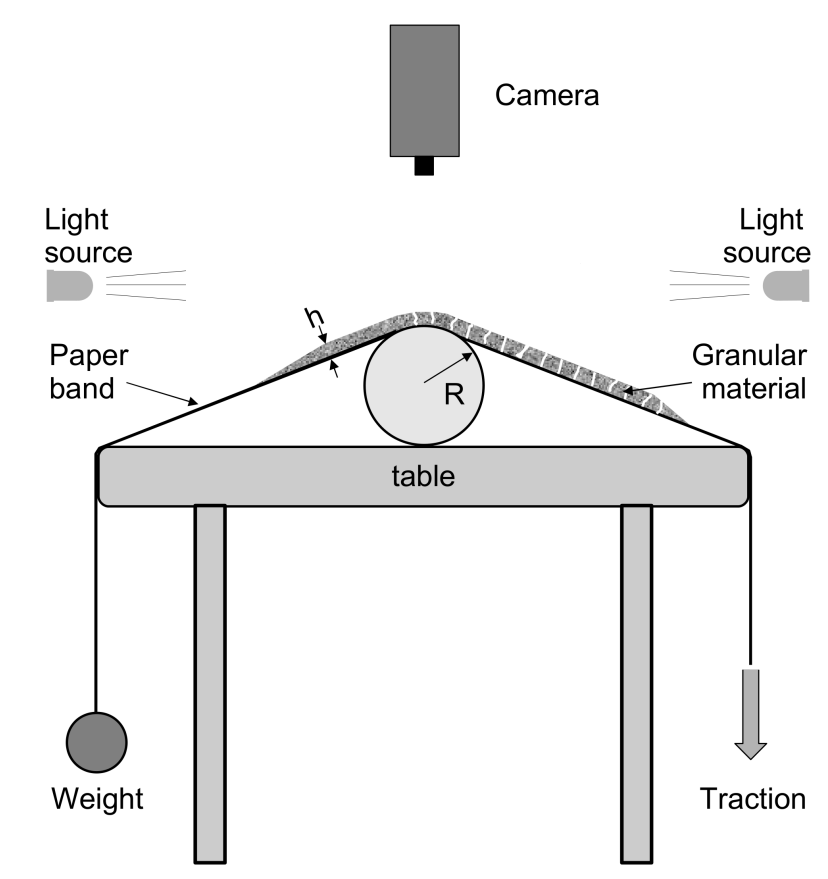

FIG. 1. Sketch of the experimental setup.

is fixed at the upper surface of a table as sketched in the figure 1. Outside the table, one end of the band is clamped whereas the tension is insured by a weight at the second end. The granular layer is prepared on one side of the cylinder. By displacing the clamped end downwards, one forces the passage of the band above the cylinder, such that a radius of curvature $R$ (from 4 to $10 \mathrm{~mm}$ ) is locally imposed to the granular layer.

The granular layer is prepared by pouring grains onto the membrane. The surface of the material is subsequently leveled by means of a cylindrical rod guided by lateral spacers, which achieves a well-defined thickness $h$ 


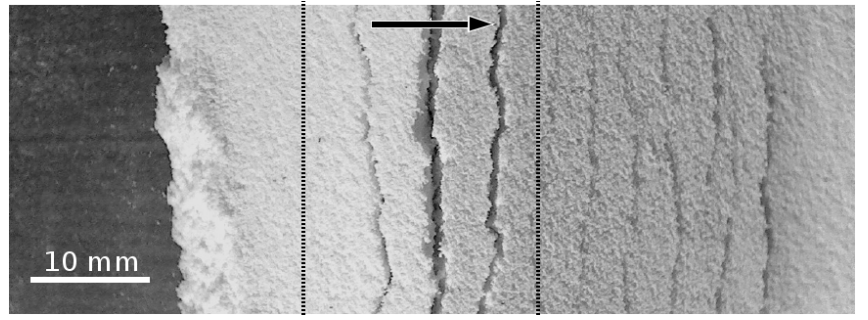

FIG. 2. Top view of the granular layer - The arrow indicates the scroll direction and the two dashed lines the cylinder, thus the region in which the bending of the material is imposed ( $h=4 \mathrm{~mm}, R=10 \mathrm{~mm}$, flour).

(1.5 to $5.0 \mathrm{~mm}$, to within $0.5 \mathrm{~mm}$ ). The granular material consists of flour or spherical glass-beads (USF Matrasur, sodosilicate glass). We shall report results obtained for various samples in a large range of bead diameters $d(0-45,45-63,63-80,80-100 \mu \mathrm{m})$, sufficiently small for the material to exhibit a significant cohesion which is, in addition, accounted for by measuring the angle of avalanche, $\theta_{a}$, in the same experimental conditions.

The free surface of the layer above the cylinder is imaged from top by means of a digital camera (KonicaMinolta, A200). Two linear light-sources (home-made arrays of LEDs) placed on the sides, about $20 \mathrm{~cm}$ away from the sample, a few centimeters above the table plane, provide a good contrast when the upper surface of the material is deformed.

\section{RESULTS}

When the granular layer is pulled above the cylinder, one observes the formation and the scroll of successive fractures in the field of the camera (Fig. 2). We estimate the average distance between two sucessive fractures, or wavelength, $\lambda$, by counting the number of fractures that passed the apex of the cylinder for a given pulling distance (The resulting accuracy is of about $0.3 \mathrm{~mm}$ ).

Let us first discuss, the role played by the radius of curvature $R$ imposed to the granular layer. First, we observe that the wavelength $\lambda$ does not significantly depend on $R$ (Fig. 3). The result could be surprizing at first sight if one condiders that the bending deformation is necessary to the formation of the fracture pattern. However, considering that the grains do not slide on the band, the imposed deformation consists of a pure bending, without stretching, at the contact with the substrate whereas it results in a stretching of the material above. The relative elongation of the free surface, $\theta \simeq h / R$, thus controls the intensity of the deformation imposed to the granular layer. A minimal $\theta$ is necessary for the fracture pattern to be observed but, similarly to what is observed when a uniaxial stretching is imposed [3], the wavelength $\lambda$ does not depend on $\theta$.

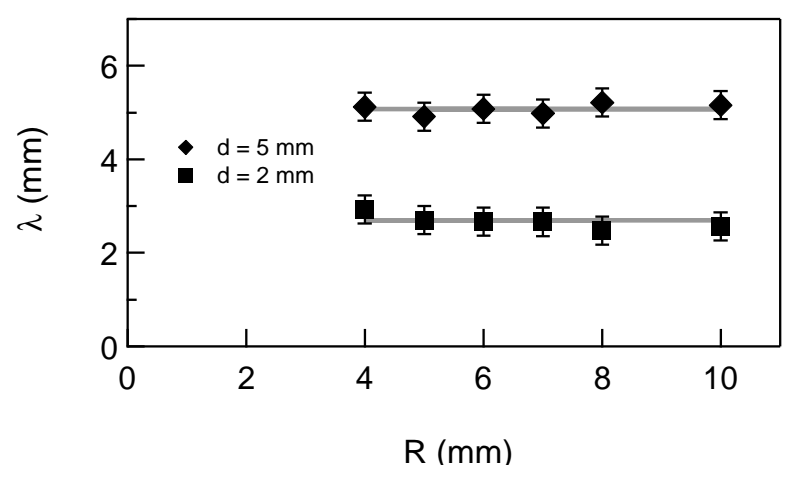

FIG. 3. Wavelength $\lambda$ vs. radius $R$ - The wavelength does not depend on the imposed radius of curvature and increases with the thickness $h$ of the layer (flour).

At this point, it is interesting to consider the dependency of the wavelength, $\lambda$, on the layer thickness, $h$. We observe that for a given material, $\lambda$ is almost proportional to $h$ (Fig. 4). The conclusion holds true as long as the thickness is not too large (typically smaller than $5 \mathrm{~mm}$ ). For larger $h$, the granular material between two fractures is likely to split in two (Fig. 4, inset), leading to a smaller average $\lambda$.

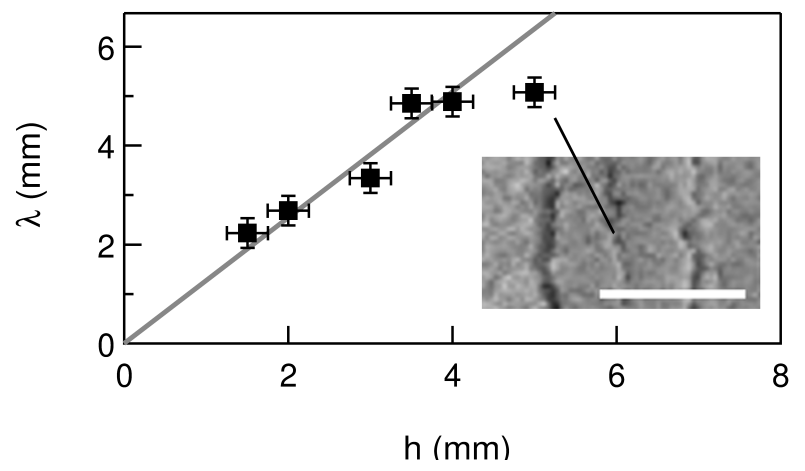

FIG. 4. Wavelength $\lambda$ vs. thickness $h$ - The wavelength $\lambda$ scales with the thickness $h$ of the granular layer. For flour, we get $\lambda=(1.25 \pm 0.05) h$. However, for the thickest layer, a secondary instability leads to the fracturing of the domains between the principal fractures and, thus, to a decrease of the average spacing between the fractures. Inset: photograph illustrating the secondary fracturing of the material between two fractures for $h=5 \mathrm{~mm}(R=7 \mathrm{~mm}$, flour $)$.

Finally, we consider the dependency of the wavelength, $\lambda$, on the cohesion which we account for by measuring the angle of avalanche $\theta_{a}$. Interestingly, we observe that, for the glass beads, $\lambda$ does not significantly depends on $\theta_{a}$ whereas the wavelength measured for flour is significantly larger. The results obtained for the glass beads are in qualitative agreements with the results previously reported for the uniaxial stretching in which $\lambda$ was ob- 
served to be independent of the grain size $d$, even if the latter are associated with different $\theta_{a}$ at a given relative humidity $R_{H}$.

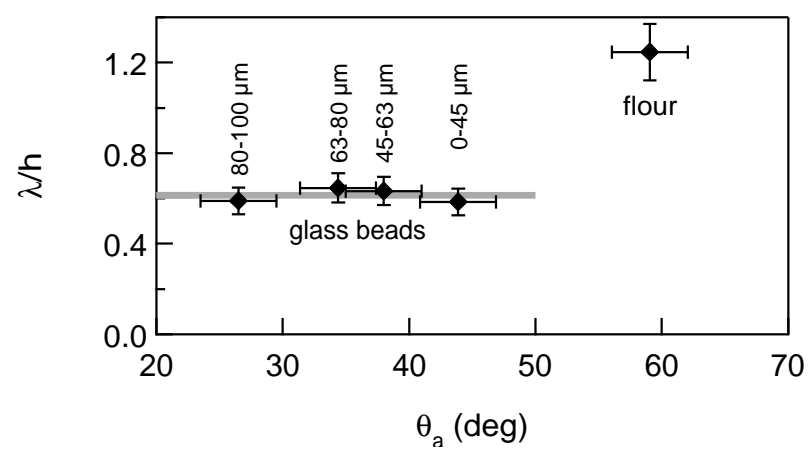

FIG. 5. Wavelength $\lambda$ vs. avalanche angle $\theta_{a}-$ For the glass beads, the wavelength $\lambda$ does not depend on the avalanche angle $\theta_{a}$, which is varied by changing the grain size $d$ at a given relative humidity $R_{H}$. By contrast, for the same $R_{H}, \lambda$ is larger for flour which exhibits a larger $\theta_{a}(R=7 \mathrm{~mm}$, $h=3.5 \mathrm{~mm}, R_{H} \simeq 35 \%$ ).

\section{THEORETICAL ANALYSIS}

In response to the overall stretch, due to the "strain softening" of the cohesive granular matter, the system spontaneously tends to modulate the deformation. We reproduce here the same reasonning as in reference [3], considering now the peculiar geometry of the flexural deformation.

Let us assume that the normal stress along the $x$-axis (Fig. 6), $\sigma_{x x}$, decreases linearly with the uniaxial strain $u_{x x}$, according to $\sigma_{x x}=\sigma_{s}\left(1-u_{x x} / \theta_{m}\right)$ when the material is stretched $\left(u_{x x}>0\right)$. For $u_{x x}>\theta_{m}$, the material breaks apart. For the sake of simplicity, the contribution of the shear is accounted for by a simple shear modulus $G$ and, in this framework, the shear stress $\sigma_{x z}=G u_{x z}$. We also assume that the grains remain in contact along the vertical so that the dilation in the vertical direction $u_{z z}$ does not contribute to any additional energetic cost. At last, seeking for simplicity, we impose that the deformation of the material does not induce any dilation so that $u_{x x}+u_{z z}=0$.

We consider a sinusoidal perturbation (wavelength $\lambda=$ $2 \pi / k$ ) of the displacement field induced by the bending deformation such that $u_{x}=x z / R+f^{\prime}(z) \sin k x$ and $u_{z}=-\left(x^{2}+z^{2}\right) / 2 R-k f(z) \sin k x$. Writing the relation imposed by the mechanical equilibrium in the horizontal plane $\frac{\partial \sigma_{x x}}{\partial x}+\frac{\partial \sigma_{x z}}{\partial z}=0$ and the conditions that $u_{x}$ and $u_{z}$ do not depend on $x$ at the contact with the substrate $\left[f(0)=0\right.$ and $\left.f^{\prime}(0)=0\right]$, we get $f(z)=a(1-\cos \omega z)$ with $k a$ the amplitude of the vertical displacement at $z=h, \omega^{2}=k^{2}(1+\xi)$ and $\xi \equiv 2 \sigma_{s} /\left(G \theta_{m}\right)$. Writing that the shear stress $\sigma_{x z}$ vanishes at the free surface

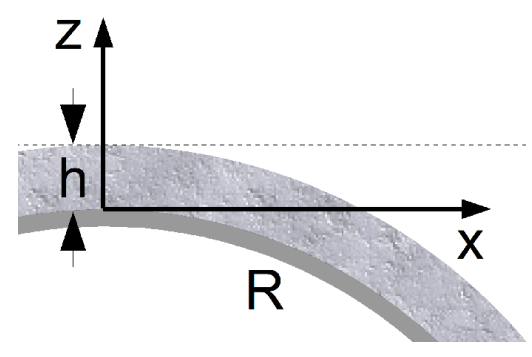

FIG. 6. Definition of the frame of reference - The deformation of the membrane is described in a cartesian frame of reference $(\mathrm{x}, \mathrm{z})$, the origin at substrate.

and, thus, that $u_{x z}=0$ for $z=h$, we get the relation $1+\xi \cos \omega h=0$, which imposes the wavelength, $\lambda$. We get as in Ref. [3]:

$$
\lambda=2 \pi \frac{\sqrt{1+\xi}}{\arccos (-1 / \xi)} h
$$

provided that $\xi \geq 1$. One can check that in the accessible range of $\xi$, the energy is a decreasing function of the amplitude $a$, whatever the strain $\theta$. Thus, the layer is always unstable provided that the decrease in the tensile stress is large enough compared to the shear modulus, i.e., $\frac{\sigma_{s}}{\theta_{m}} \geq \frac{G}{2}$. The growth of the instability is limited by the condition that $u_{x x} \geq 0$ for all $x$ and $z=h$ (the strain in the plane $z=0$ does not lead to any compression of the free surface), so that $\theta-a \omega k \sin \omega h=0$. Thus, the amplitude, $k a$, of the vertical displacement is predicted to scale with $h^{2}$.

\section{DISCUSSION AND CONCLUSIONS}

In Ref. [3], we reported for the first time the destabilization process of a material exhibiting a negative stressstrain sensitivity, $d \sigma / d u_{x x}<0$, when subjected to uniaxial strain. In the present Brief Report, we complemented the previous study with experimental results obtained in the case of a pure flexural deformation.

In the latter experimental situation, the amplitude of the imposed deformation is given by the ratio $h / R$ of the layer thickness $h$ to the imposed radius of curvature $R$. As expected from simple theoretical arguments, the wavelength $\lambda$ does not depend on $R$ and scales with the layer thickness $h$. Quantitatively, $\lambda$ is similar to that measured for pure stretching for the same material and same layer thickness, $h$. Note, however, that the amplitude of the deformation is expected to scale with $h^{2}$ for the flexural deformation, where it scales with $h$ for pure stretching.

Interestingly, even if controlled by the cohesion, the wavelength $\lambda$ does not exhibit any sensitivity to the angle of avalanche $\theta_{a}$ achieved, for a given relative humidity $R_{H}$, by changing the grain size $d$. The same conclusion 
was drawn from the results of the pure stretching, which also pointed out, for a given grain size $d$, an increase of $\lambda$ with $R_{H}$. We herein obtained that, for given thickness $h$ and relative humidity $R_{H}$, a different wavelength $\lambda$ can be achieved by changing the properties of the grains the material is made of.

A complete understanding of the instability surely de- serves further investigation and we are now focusing on the third pure deformation, the shear, which can be applied to the granular layer and on direct measurements of the parameters of the material (shear modulus $G$, tensile stress $\sigma_{c}$, maximum stretching $\theta_{m}$, etc.).

The authors acknowledge the financial support from the contract ANR-09-BLAN-0389-01 (CONICYT 011).
[1] J. Crassous, E. Charlaix, and J. L. Loubet, Phys. Rev. Lett. 78, 2425 (1997).

[2] C. D. Willett, M. J. Adams, S. A. Johnson, and J. P. K. Seville, Langmuir 16, 9396 (2000).
[3] H. Alarcon, O. Ramos, L. Vanel, F. Vittoz, F. Melo and J.-C. Géminard, Phys. Rev. Lett. 105, (2010) 208001. 